\title{
EILEEN GRAY: LA EMANCIPACIÓN FEMENINA EN LA ARQUITECTURA MODERNA
}

\section{EILEEN GRAY: WOMEN'S EMANCIPATION IN MODERN ARCHITECTURE}

\author{
David Luís López \\ Universitat Jaume I de Castellón
}

\section{RESUMEN}

Eileen Gray fue una mujer pionera en desarrollar su carrera dentro de una profesión liberal, y lo hizo en diferentes campos, como el diseño, el interiorismo o la arquitectura. Su trayectoria vital se convierte en una narración ejemplar de la emancipación de la mujer en el siglo XX. A pesar de las muchas dificultades a las que tuvo que enfrentarse, logró establecer un lenguaje propio en un periodo convulso como lo fueron las primeras décadas del siglo pasado, bajo la premisa de que la forma debía seguir a la función, aunque siempre manteniendo una composición que fuera estética. Algunos de sus diseños de mobiliario, han sido en incluidos en numerosas ocasiones dentro del Art Déco, pero del mismo modo que ocurriría con su arquitectura-que se vería influenciada necesariamente por los grandes arquitectos del Movimiento Moderno- su propio lenguaje acabaría por imponerse; un lenguaje siempre inconformista e inmerso en una constante evolución hacia esa adaptación de los espacios para las personas.

Sus trabajos y su capacidad emprendedora hicieron de Eileen Gray una mujer profesional en un eminente campo dominado por lo masculino. Consecuentemente, muchas serían las barreras propias de las estructuras patriarcales que debería superar para desarrollar su carrera profesional. A pesar de todo, consiguió enfrentarse a ellas y superarlas, posiblemente por su actitud abierta y creativa, amante del conocimiento y con unas ideas progresistas gracias a las que consideraba que todos sus pasos eran una forma de colaborar en la construcción de una nueva sociedad, en definitiva, de ese Hombre -y también mujer- Nuevo del que nos habla el reconocido arquitecto de este periodo, Le Corbusier.

Posiblemente, la construcción de la Villa E1027 en Cap Martin fue una de las tareas más ambiciosas de su trayectoria, pero también fue, sin duda, uno de los episodios más traumáticos de la biografía de Gray, y que indiscutiblemente tiene grandes connotaciones de género, en parte porque la legalidad impone sus propias reglas, y en parte porque la moral patriarcal de la sociedad continuaba siendo demasiado rígida para permitir que se cumpliera la voluntad de una mujer, por legítima que esta pudiera llegar ser.

Palabras clave: arquitectura, historia, género, estética, arte, diseño.

\section{ABSTRACT}

Eileen Gray was a pioneer in developing her career within a liberal profession, and did so in different fields, such as design, interior design and architecture. Her life has become a unique account of the 
women's emancipation in the twentieth century. In spite of the many difficulties that she had to face, she managed to establish her own language in a tumultuous period, the first decades of the last century, under the premise that form should follow function, although always maintaining that works should have aesthetic properties. Some of her furniture designs have been identified in several occasions as Art Deco, but just as would happen with her architecture - which was obviously influenced by the great architects of the Modern Movement - her own language would eventually be imposed; a language which was always nonconformist and immersed in a constant evolution towards that adaptation of the spaces for the people.

Her work and entrepreneurship made Eileen Gray a professional woman in a male-dominated field. Consequently, she had to overcome many barriers of patriarchal structures to develop her professional career. Despite all troubles, she managed to face them and overcome them, possibly because of her open and creative attitude, her thirst for knowledge and progressive ideas. She considered that all her steps were a way of collaborating in the construction of a new society. In short, she contributed to the creation of that New Man (and Woman) envisioned by the renowned architect of this period, Le Corbusier.

Possibly, building Villa E1027 in Cap Martin was one of the most ambitious tasks of her career, but it was also undoubtedly one of the most traumatic episodes in Gray's biography. It undoubtedly has major gender connotations, on the one hand because legality imposes its own rules, and on the other hand because the patriarchal society values continued to be too rigid to allow a woman's will to be fulfilled, however legitimate it might be.

Keywords: architecture, history, gender studies, aesthetics, art, design.

Las primeras décadas del siglo XX serían convulsas para la sociedad europea, que experimentaría en primera persona la devastación del viejo continente tras la Gran Guerra. En ese contexto resultaría complicado encontrar mujeres arquitectas, y es que, tal y como apunta Rita Levi-Montalcini «en el siglo pasado y en las primeras décadas del nuestro, dos cromosomas $X$ constituían una barrera insuperable para entrar en las escuelas superiores $y$ realizar los propios talentos» (Levi-Montalcini, 1989: 36 cit. en Espegel, 2007: 79-80).

Sin embargo, y a pesar de todos los problemas sociales y económicos, será un periodo donde las manifestaciones culturales, artísticas y arquitectónicas proliferan como nunca. Precisamente en arquitectura aparecen nuevos materiales, y también, nuevas formas de concebir sus usos, hasta entonces muy restringidos. Es también el periodo del Diseño Industrial; por primera vez la historia del arte y la elaboración de productos siguen patrones diferentes. La forma, en el diseño, necesariamente debe adaptarse y responder a la funcionalidad que 140 es, en definitiva, el último fin para el que cada objeto de diseño está destinado. 
En este contexto, nos encontramos ante una arquitecta de gran personalidad, y que sería sin duda, precursora de muchas otras mujeres que se adentrarían en el ámbito del diseño y la arquitectura. Pocas mujeres han logrado tener un reconocimiento suficiente, al menos durante este periodo, para que sus trabajos y sus biografías hayan llegado hasta nosotros. $\bigcirc$ al menos, que nos hayan llegado desde esa perspectiva de la individualidad que caracteriza el trabajo y el propio desarrollo vital de Eileen Gray (Fig. 1). Uno de los mejores retratos que se conservan de esta arquitecta es el que realizara la fotógrafa Berenice Abbott, quien fuera colaboradora de May Ray, y que posteriormente se convirtió en discípula de Eugène Atget. Tras la muerte de éste, Abbott se trasladaría a Estados Unidos, donde consiguió ser la primera mujer capaz de fotografiar Nueva York con ojos modernos, logrando cosechar un gran éxito con su serie Changing New York. Esta serie la convertiría en una de las mejores fotógrafas de la arquitectura contemporánea. En sus imágenes:

... se puede distinguir una mirada surrealista sobre la ciudad, una mirada repleta de aportaciones simbólicas sobre la nueva era industrial y la desorientación de la vida en la ciudad, donde objetos y personas se confunden como tal» (Campelo, 2012: 249).

Nacida en Irlanda, el 9 Agosto de 1878, Eileen Gray creció en una familia aristócrata escoceso-irlandesa, y vivió durante su niñez en dos lugares, por una parte en la residencia familiar de Irlanda, y por otra en la casa materna de Londres. Sus padres convivieron durante un tiempo sin apenas dirigirse la palabra, algo que acabaría con el regreso de su padre a Italia, su país de origen. Así lo explica Peter Adam, el biógrafo oficial de Gray, «She remembered them sitting silently at either end of the longo dining-room table. But hen her father went back to ltaly, and except for a few visits, remained there for the rest of his life» (1987: 12). Tal vez, esta situación de problemas de pareja influiría sobre Eileen más de lo que muchos han pensado, de manera que ella nunca querría mantener una relación formal de matrimonio con nadie a lo largo de su vida.

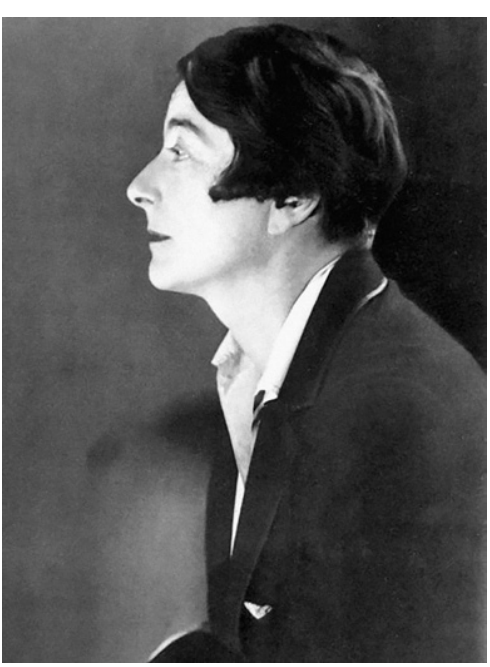

Fig. 1. Retrato de Eileen Gray realizado por Berenice Abbot en Paris, 1926.

Gray llegó a considerar a cada uno de sus progenitores de una forma bien diferente; su madre representaba para ella la autoridad, una mujer que «had a rather solemn face, as if she rarely smiled; a woman whose pride was hurt through the loss of the love of her husband» (Adam, 1987: 12). Mientras tanto, la figura de su padre tenía otras connotaciones para ella: 
«If her mother instilled in Eileen good manners and a feeling for social propriety, her father taught her the love of freedom» (Adam, 1987: 12). Tal vez la forma de entender la vida de su padre (alrededor de la profesión pintor, con su aura de bohemia) inspiró a Eileen unos valores que, aunque se adaptaban en gran medida a la moral victoriana de la Inglaterra de finales del siglo XIX, no eran del todo convencionales ni extremadamente estrictos. Como tampoco sería convencional la situación familiar, pues en ese periodo la separación matrimonial no era algo común, y muy pocos matrimonios, a pesar de sus desavenencias daban ese paso. Algo que, sin duda, también tiene unas connotaciones progresistas en las relaciones de género si tal separación sitúa a los dos cónyuges en una posición igualitaria.

El carácter independiente de Eileen Gray la llevó a estudiar en la escuela de arte Slade School of London, donde conoció a grandes amigos - a pesar de su carácter tímido, que en demasiadas ocasiones se le había considerado como una actitud de superioridad, que nada se correspondía con la realidad-con los que compartía un mismo sueño: viajar a París para continuar sus estudios y desarrollarse profesionalmente, y donde, sin que ella lo imaginara, llegarían a encontrarse en un futuro. Sin embargo, las circunstancias no posibilitaron que aquel sueño que anhelaba fuera el siguiente episodio de su vida, resultando que la primera vez que viajó a París, lo hizo acompañada de su madre en el año 1900. Aquella capital europea cautivaría a esta arquitecta para siempre. «Eileen was impressed with the generosity of the street plan of Paris, with the width of the leafy boulevards, the elegance of the shops, and the goings-on in the street, where electricity was replacing gas lamps» (Adam, 1987: 25).

Cuando tuvo que regresar a Londres, comprendió que debía trazar un plan para regresar a aquella ciudad, París, y poder ampliar allí sus estudios. De esta manera, con la ayuda de sus amigas Kathleen Bruce y Jessie Gavin, lograría convencer a su madre y regresar a París. Hay que recordar que el pensamiento de Eileen Gray tiene mucho que ver con el de autores coetáneos anglo-irlandeses. Como explica Carmen Espegel:

Eileen Gray representa en la arquitectura, el homólogo coetáneo de aquellos escritores 0 artistas anglo-irlandeses que, al igual que sus compatriotas James Joyce y Samuel Beckett, optaron por el camino del exilio cultural. Común a todos ellos fue su afán por dinamitar la mórbida rigidez de la moral victoriana reivindicando lo emocional, lo vital, lo sensitivo, a través de la nueva razón (2007: 101).

Su llegada se produce en el año 1902, pero tres años más tarde tiene que abandonar la ciudad, por haber contraído tifus, y convalecerá de esta enfermedad en Argelia, donde «entra en contacto con la arquitectura mediterránea que tanto influirá en los jóvenes arquitectos 
modernos» (Espegel, 2007: 101). Unos meses después, ya recuperada, viajará a Londres, para visitar a su madre con problemas de salud. Allí, casi por casualidad, descubrirá la tienda de D. Charles, e inmediatamente «She walked into Charles's shop and inquired if she could work there for a while» (Adam, 1987: 49), tal vez sin imaginar que este sería un momento clave en su carrera profesional porque descubriría las posibilidades que el lacado podía ofrecer para el diseño y la restauración.

A su regreso se establecería en un apartamento en la Rue Bonaparte, una casa que curiosamente conservaría hasta el final de sus días. La ciudad se encontraba en periodo de grandes cambios, y en ella comenzaban a trazarse las líneas maestras de un estilo tan relevante como interesante, el Art Déco, que sin duda, influiría en su estilo personal para elaborar sus diseños. Gray se sentía, a pesar de su carácter tímido, muy cómoda en el ambiente bohemio y en el círculo artístico donde se movía. Su estilo de vida era el de muchos jóvenes de su época, espíritus libres y emancipados. Más difícil era para las mujeres, pero lo podían lograr, al menos de forma relativa, siempre y cuando, eso sí, pertenecieran a una determinada clase social. La libertad para ellas era un privilegio de clase.

Es precisamente en este momento, a su regreso de Londres, cuando conoce al que sería su verdadero maestro en la técnica del lacado, Seizo Sougawara, un joven japonés, que había crecido en una de las regiones con más tradición en el uso de esta técnica y los elementos decorativos de su país. Gracias a su ayuda, Gray logró perfeccionar su técnica siendo capaz de:

... decorar amplias e impecables superficies, experimentando con metal, incrustaciones de madreperla y bajorrelieves y logra extender la paleta cromática más allá de los tradicionales tonos negros, bermellones y marrones para conseguir azules y verdes profundos (Espegel, 2007: 101).

Mediante el uso de este producto, Gray logra ganarse la vida con el diseño y venta de objetos lacados, y comienza a producir platos decorativos, cuencos, y otros objetos, entre los que muy especialmente destacan sus biombos. Un objeto predilecto, que posteriormente en su arquitectura, servirá para delimitar pequeños espacios en grandes superficies diáfanas, abiertas y transitables, pero que tan bien sabe complementar Gray con la exacta dosis de intimidad a la que, significativamente, contribuyen estos objetos, que acabarían siendo elementos que identificaban la mayoría de sus trabajos. Su obra fue expuesta en el VIII Salon des Artistes Décorateurs, sin duda una buena muestra de la importancia que sus diseños adquirieron en el París de aquél momento.

Durante 1914, en pleno contexto bélico, Gray construyó el biombo Le Destin (Fig. 2), un encargo que le había hecho un buen cliente suyo, Jacques Doucet. Esta pieza, acabada: 
... con laca de color rojo oscuro sorprende por el empleo de estilos completamente distintos en cada uno de los lados. A pesar de que ambas reproducciones son bidimensionales y tienen la misma tonalidad, parecen señalar hacia dos tendencias artísticas muy distintas (Hecker, 1993: 13).

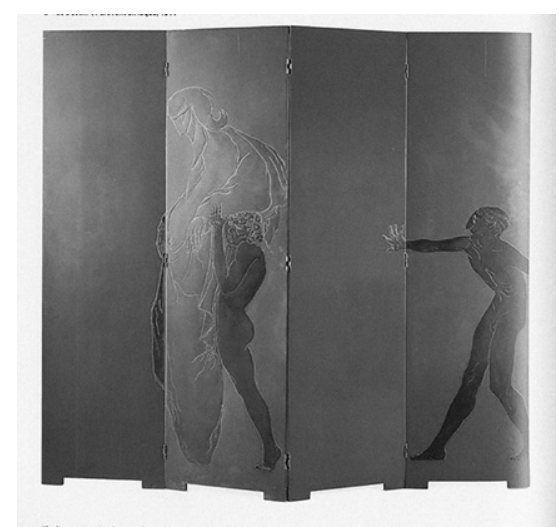

Fig. 2. Eileen Gray. Le Destin, biombo de cuatro paneles, 1913.

Será su último trabajo antes de regresar a Londres, huyendo de la Guerra durante dos años. Allí seguirá trabajando en diseños con lacados, siempre acompañada de Sougawara, aunque con escaso éxito comercial en una sociedad -la inglesa- en la que la moral y los estilos tradicionales se imponían ante cualquier atisbo de modernidad y de cambio.

Cuando regresa a París tras el conflicto, recibe su primer encargo global. Una rehabilitación integral en la que, podrá establecer esa primera relación con la arquitectura, -aunque no en su totalidad por tratarse de una reforma. En este espacio, en la Rue de Lota:

Su innovación más interesante fue el uso de unos biombos de bloques lacados en negro utilizados para transformar el largo hall de entrada donde formaliza una combinación entre escultura, arquitectura y mobiliario (Espegel, 2007: 102).

Eileen Gray pretendía con esta reforma poder conjugar diseño, arquitectura y creatividad, siempre secundando el principio de la función. Por ello, cada una de las piezas que conformaban el interior del apartamento, fue cuidadosamente diseñada específicamente para el lugar que debía ocupar. Gray consiguió crear un ambiente que se alejaba de lo habitual en aquel momento, y poder reinterpretar los espacios y las funciones que estos debían cumplir. En definitiva, «todos los objetos fueron subordinados a una idea superior» (Hecker, 
1993: 20). Toda esta reforma, como hemos advertido, fue muy importante en su carrera, puesto que le brindaría la oportunidad de realizar una intervención total, sin embargo, «el diseño más sorprendente de este apartamento fue el sofá piragua» (Fig. 3) (Dachs, 2013: 28), una pieza que ha caracterizado a esta diseñadora y que la vincularía al movimiento Déco, aun existiendo diferencias que se acentuarían con el desarrollo de su carrera. Esta chaise longue «combinaba texturas de la laca color marrón del exterior y la plata del interior y era sostenida por doce pequeños arcos» (Dachs, 2013: 28).

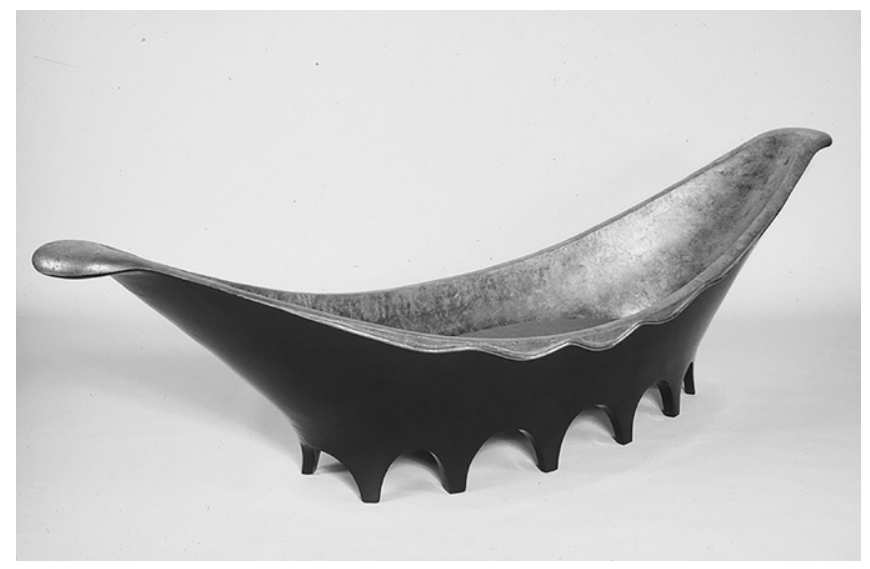

Fig. 3. Eileen Gray, sofa piragua o Canoe, 1921.

La reforma del apartamento Lota, significó para la carrera profesional de Eileen Gray una apertura de nuevas posibilidades para desarrollar su talento. A los pocos años, tras surgir la necesidad de un espacio en el que mostrar sus productos-alfombras, muebles y objetos- decide abrir la Galería Jean Désert, en mayo de 1922. Sin duda, uno de los momentos más apasionantes de su trayectoria como diseñadora, el establecimiento de un espacio comercial abierto a un público, posiblemente menos histriónico, evitando enfocar su trabajo exclusivamente para una reducida élite económica. En ese sentido, pretende redirigir sus diseños hacia modelos menos asociados con la frivolidad, la riqueza y con el más absoluto de los individualismos por tratarse de piezas que, generalmente, no se repetían.

Posiblemente inspirada por la famosa lectura Ornamento y Delito, de Adolf Loos, encamina sus diseños hacia esa producción más económica a la que nos referimos, mediante el uso de materiales menos exclusivos, más generalistas. Materiales de procedencia industrial que comenzaban en este periodo a aplicarse en el diseño de objetos para el uso cotidiano, 
con formas más limpias y remates más sencillos. Así, Eileen Gray «comenzó a diseñar objetos para producir en serie; mobiliario que creía que llegaría a cambiar el estilo de vida de la sociedad» (Dachs, 2013: 44).

Uno de los errores más habituales durante este periodo, en el ámbito del diseño del mueble, resultó ser que, mediante esa huida de la forma en búsqueda de la funcionalidad más pura, algunos diseñadores dejaron de lado los valores estéticos del mobiliario, pensando que estos eran irrelevantes, y que todo se regía únicamente por la función que cada objeto debía cumplir. Es por ello, que «el logro de sus diseños [de Eileen Gray] radicaba en que no subordinaba el valor artístico al funcionalismo, sino que fusionaba ambos conceptos» (Dachs, 2013: 44), logrando cumplir, por tanto, la premisa más importante del diseño industrial: conjugar función y forma con la mejor disposición estética posible, de manera que «algo impráctico jamás puede ser bello» (Wagner cit. en Hofmann, 1992: 302).

Bajo estas premisas, Gray consigue abrir Jean Désert en un local ubicado en el número 217 de la comercial calle Rue du Faubourg Saint-Honore. Un espacio en el que tuvo que intervenir para poder ubicar en él su espacio de trabajo y una selecta exposición de sus diseños -y también de otros artistas como Chana Orloff y Ossip Zadkine- de acuerdo con ese estilo arquitectónico interior que ya había descubierto en trabajos como el del apartamento Lota. De este modo:

For her new gallery, Gray designed a simple but striking façade. Beneath elaborate sculptured baroque stonework on the first floor, she drew a very simple grid incorporating large areas of window and doors, with chequered panels reemphasising the strict geometry of the elevation (Garner, 1993: 24).

Pero las intervenciones sobre este local no quedarían sólo en su fachada, también dentro experimentaría grandes cambios con la llegada de Gray; «inside she ripped out the moldings and fittings and painted the walls all white. She put in a new modern staircase to the basement» (Adam, 1987: 119). Eileen Gray fue una mujer cuyo trabajo era sinónimo de calidad y perfección. Algo que no podría haber sido de tal forma de no contar con esa gran dedicación y la pasión por el trabajo que la caracterizaba. Es por ello que sus obras integrales gozaban de una armonía que impregnaba todo, seguramente porque todos los diseños respondían a esa concreción en la que se encontraban y para la que se encontraban. Con esta forma de trabajar y de pensar, no es de extrañar que las obras que realizaría sobre su nueva tienda, la galería Jean Désert, serían modificaciones que, aun no siendo grandes intervenciones arquitectónicas, darían un giro absoluto al espacio anterior. La nueva galería, con su nuevo diseño, estaba preparada para ser el epicentro de una nueva forma de diseñar que sería reconocida por los principales 146 creadores y críticos europeos. 
Tras la apertura de este espacio, Eileen Gray participará en diferentes exhibiciones y encuentros por toda Europa, donde cabe destacar el Salon des Artistes Décorateurs. Pero sería en una exhibición en Ámsterdam donde sus creaciones llamarían la atención de uno de los miembros del grupo De Stijl. Este grupo se caracterizaba por entender los conceptos de arte, arquitectura y diseño en su globalidad, con sus innegables interconexiones que evidencian esa visión de obra de arte total sobre la que trabajaban, reflexionaban y discutían, siempre defendiendo unas dinámicas y pautas elementales que eran comunes en todos sus trabajos, las formas geométricas y las superficies cromáticas planas en colores primarios. Al respecto Garner explica que:

Her work atracted the attention of Jan Wils, who entered into correspndence with her [...]. Wills was a member of the De Stijl group, and it was in homage to the ideals of this group that Gray designed a remarkable table at this time. (1993: 27).

Sería justamente a través de estos contactos, y la asistencia a este tipo de eventos, gracias a lo que tomará conciencia de la importancia de la arquitectura como base elemental del resto de artes aplicadas. Esta es una de las premisas base del Movimiento Moderno que comenzaba a emerger en este momento. También conocería al crítico de arquitectura, Jean Badovici, quien animaría a Gray a adentrarse en la arquitectura por sus aptitudes, y con quien, además, acabaría teniendo una relación sentimental, a pesar de la diferencia de edad de casi veinte años en un contexto en el que, salvo contadas excepciones, la mujer nunca era mayor que el hombre.

Jean Badovici estudió arquitectura en l'Ecole Supérieure d'Architecture, pero nunca ejerció como tal, sino que encaminó su carrera profesional hacia la crítica de la arquitectura, la edición y la elaboración de diferentes revistas sobre arquitectura. Era unos veinte años más joven que Eileen Gray, pero ambos descubrieron en cada uno de ellos algo que les uniría durante bastante tiempo. Ella, tal vez veía en él las ganas, el entusiasmo, la juventud y la frescura de la ilusión por hacer grandes cosas. Él, posiblemente, veía en ella una diseñadora con grandes aptitudes para poder desarrollar sus ideas -incluso las sugerencias de él mismo como crítico- en el campo de la arquitectura, y suficiente capacidad financiera para ponerlos en práctica.

La realidad es que Eileen Gray conoció, gracias a Badovici, a los principales arquitectos del Movimiento Moderno, merced a que muchos de los trabajos y entrevistas publicados por Badovici giraban en torno a las más recientes e interesantes construcciones de estos arquitectos, entre los que cabe destacar a Le Corbusier, por la relación personal -que posteriormente ampliaremos- que mantuvo con la pareja. Una de estas revistas, en la que 
trabajó Badovici, sería L'Architecture Vivante, y gozaría de gran éxito pese a ser una revista especializada, tanto es así que al poco tiempo este magazine:

... amplía su apertura internacional, y comienza a publicar los «iconos» de la vanguardia: proyectos de Mies van der Rohe, van Doesburg, van Eesteren, Rietveld [...] política que se confirma en los números siguientes, y se transformará en una de las colecciones más sistemáticas de la «nueva arquitectura internacional» (Espegel, 2007: 107).

Dentro de este contexto de auge arquitectónico e intelectual, Badovici propone a Eileen Gray la construcción de una residencia para descansar, para pasar largas temporadas estivales en la rivera del sur de Francia, y anima a la arquitecta a buscar el mejor lugar para desarrollar el proyecto. Un proyecto cuya «idea sería proyectar, construir y amueblar una casa que fuera un modelo pionero, un experimento intensamente personal en el espíritu progresista del Movimiento Moderno» (Espegel, 200: 107).

Es en este periodo, conocido como un periodo de auge económico de entreguerras, cuando determinados lugares -como Saint Tropez o Montercarlo- se convierten en un destino turístico por primera vez en la historia. La combinación de ocio y descanso convierte estos destinos de la costa del mediterráneo en el lugar perfecto para personas de toda Francia que tratan de escapar de la alterada vida de la gran ciudad. Gray visitó varias veces la costa del sur de Francia y llegó a pensar que cualquier lugar cercano al mar sería perfecto para poder descansar. Con estas premisas, encontró un terreno ubicado en Cap Martin-Roquebrune que resultaría ideal para desarrollar su proyecto más personal. Ubicado a escasos metros del mar, era un sitio privilegiado, en el que este hecho condiciona todo el conjunto arquitectónico, de manera que «el carácter marítimo de la vivienda emergía,
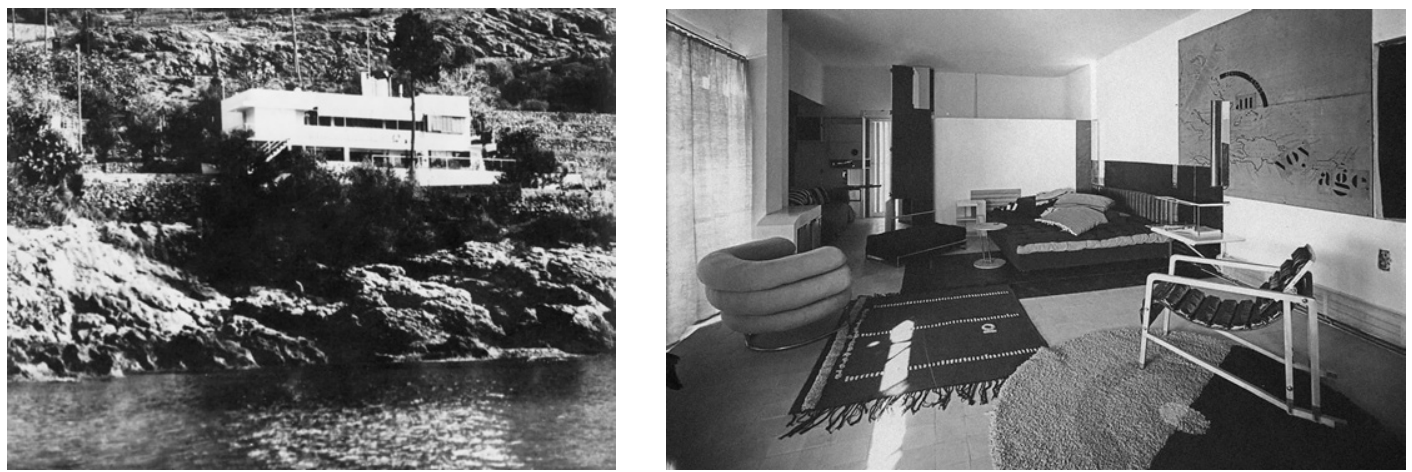

Fig 4. Eileen Gray, Exterior e Interior de Maison en bord de mer o E1027, Cap-Martin Roquebrune, 1927. 
inevitablemente, del ambiente, de los materiales impuestos por dicho ambiente, y de la vecindad del mar» (Gray cit. en Hecker, 1993: 60).

La historia de la villa E1027, la que construye en Cap Martin-Roquebrune (Fig. 4), es otro de los episodios más convulsos de la vida de la arquitecta. Bien por tratarse de un proyecto personal que realiza apoyada por Badovici -con quien mantenía una relación sentimental en aquel momento en el que ella todavía no era arquitecta titulada- o por las posteriores intromisiones del propio Le Corbusier, hacen que la E-1027 tenga una historia propia. Su propio nombre, ya en clave, nos revela la inspiración personal de la artista: la $E$, es la inicial de su nombre, mientras que el número 10, es la letra J en el alfabeto, e inicial de Jean Badovici, continúa con el número 2 es la posición de la $B$-que se corresponde con el apellido de él; y por último, el número es la posición de la $G$, también en nuestro alfabeto y tiene su correspondencia con el apellido de Eileen. Por otra parte, es importante hacer especial mención a este proyecto, pues en él se pueden observar algunos de los aspectos más importantes a los que se enfrenta Gray por su condición de mujer. Las cuestiones de género no eran menores, aun tratándose de una élite cultural de reconocido progresismo.

La E-1027 es una vivienda construida para «una persona que ama el trabajo, el deporte y recibir a sus amigos» (Gray cit. en Dachs, 2013: 54). Una casa que, como hemos apuntado, sería diseñada para ella misma y de acuerdo con su forma más personal de entender el mundo y la vida, en definitiva, de comprender sus convicciones, tal y como apunta Marcos «era su primera oportunidad de construir una obra de arquitectura con arreglo a sus convicciones más íntimas y los anhelos de diseño de una morada que lo sería para ella misma y para su amante, con quien compartía buena parte de sus ideales concernientes a la arquitectura» (2011: 263). En ese sentido, observamos como la definición arquitectónica de los espacios resultaba novedosa, inspirada en esa búsqueda de la independencia de los espacios. Respecto a esto, es importante recordar las palabras de Hecker:

Las articulaciones espaciales resultantes de los desplazamientos y la estructuración de los espacios construidos con la «piel interior» lleva a que casi cada espacio se estructure en varios «lugares». Estos «lugares» constituyen cada uno de ellos una unidad espacial propia y sirven a una función específica. Sin embargo, el espacio global puede seguir leyéndose como un todo (Hecker, 1993: 58).

Otra de las características más interesantes de la vivienda -y un hecho que a medida que han pasado los años se ha ido vinculando a una forma propia de entender la arquitectura por parte de muchas mujeres- es la capacidad del diseño exterior para entrar en sintonía con el paisaje y el clima, y a su vez con el propio interior de la vivienda. En ese sentido, 
observamos como «el ensamblaje, prácticamente completo, entre espacio interior y exterior [...] es ejemplar» (Hecker, 1993: 59). Uno de los hechos más significativos de la vivienda es que la mayoría de los muebles que componían los espacios interiores, eran piezas únicas que habían sido diseñadas específicamente para aquella casa, y para cumplir una función exacta. Además de sus famosas alfombras -que ya hemos mencionado anteriormente-, uno de los muebles más significativos, y que muestra la vertiente solidaria de la diseñadora y arquitecta, es la pequeña mesa que recibe el mismo nombre que la casa, E1027. En esta pieza, «the design combines simplicity of form and function: the stand consists of two concentric forms in tubular steel, the lower one an incomplete circle which functions as the stand and the upper one encases a circle of tempered glass which functions as the table surface» (Moran, 2013: 11). Por otra parte, la inspiración de su diseño telescópico regulable en altura, no fue otra que «to enable her sister to enjoy breakfast in bed during her visits» (Rawsthorn, 2013: $\mathrm{s} / \mathrm{p}$ ) Gray diseña un producto que facilita, en este caso a su hermana enferma, durante sus estancias en la casa, el permanecer en la cama hasta su recuperación. Esta idea del «diseño amigo» es propio de algunas diseñadoras actuales, curiosamente también de la órbita del diseño galo, como es el caso de la francesa Matali Crasset, que diseñó su conocida pieza Cuando Jim viene a París para atender a uno de sus amigos durante su estancia en la casa de la diseñadora.

Conforme a lo que habíamos advertido, tanto Eileen Gray como Jean Badovici disfrutaron de la vivienda durante unos años haciendo de ella exactamente el uso para el que fue concebida. Por aquella casa pasaron importantes personalidades de la arquitectura, entre las que cabe destacar a Christian Zervos, Claude-Roger Marx, pero muy especialmente a Le Corbusier. Éste tenía una muy buena relación con Jean Badovici gracias a la revista que dirigía. En alguna ocasión, Le Corbusier llegó a mantener correspondencia epistolar con Gray, y sería en una de esas cartas donde el afamado arquitecto confirmaría la excelencia del diseño de Gray respecto a la E-1027, considerándola una «casa llena de sentido arquitectónico» (Le Corbusier cit. en Marcos, 2011: 267). Posiblemente uno de los hechos que más llamó la atención a Le Corbusier de esta casa era que los muebles «se estructuraban en términos de prototipos para una posterior producción. El uso de elementos prefabricados en paneles, ventanas o incluso puertas subrayaban este aspecto y muestran su orientación hacia la industrialización» (Espegel, 2007: 127).

A pesar de que Eileen Gray no conseguiría obtener ningún encargo, y que siempre construyó para ella misma, elabora durante este periodo otros diseños arquitectónicos muy interesantes ajenos a esta villa, entre los que cabría destacar Petite Maison pour un ingenieur, o la reforma del apartamento de París de Badovici, un pequeño estudio de apenas cuarenta 
metros cuadrados en el que consigue «un estudio que irradia una notable amplitud, porque se confrontaron hábilmente diferentes relaciones espaciales» (Hecker, 1993: 114). En definitiva, observamos como su carrera profesional no se detiene y elabora una serie de proyectos siempre enmarcados en ese estilo moderno al que pertenece la arquitecta, pero siempre con su huella más personal. Una huella personal, que recoge elementos de proyectos ajenos y los desarrolla con sus propias ideas, en ese sentido:

... la originalidad de Gray se basa en la interpretación poética de los mejores sistemas de los mejores proyectos, de los mejores autores. Ello requiere una capacidad crítica que exige un profundo esfuerzo. Su originalidad no es aquella que pudiera denominarse «originalismo» [...] sino aquella que pudiéramos denominar «origenialidad», término utilizado por el cáustico inconformista e irónico compositor Eirk Satie, que remite a la cualidad de lo original -que en su primera acepción es la reducción al origen, a sus datos originales, auténticos y esenciales- el arquetipo sin renunciar a la historia (Espegel, 2007: 112).

Además, es también en este momento -durante 1930-, un año después de que acabara la construcción de la villa, cuando Gray decide cerrar la Galería Jean Désert. Fue una decisión difícil por parte de la arquitecta, pero el giro que habían dado sus trabajos fue lo que la llevó a tomar la decisión, a pesar de que sus nuevos diseños, con formas ya modernas y alejadas de su estilo más próximo al Déco, también tuvieron aceptación en aquél espacio comercial.

Retomando algunos de los aspectos que conciernen a la villa E1027, resulta curioso apuntar que, a pesar de las excelentes características que poseía la vivienda, y del reconocimiento que se había hecho sobre esta casa quienes la conocían, "Badovici no tenía gran interés en demostrar la capacidad creativa de Gray [en lo concerniente a la arquitectura] o en hacer saber que ambos habían construido la casa» (Espegel, 2007: 108). De hecho, no sería hasta pasados unos años cuando la propia revista que editaba la pareja de Gray haría referencia a la E-1027, y lo haría atribuyéndole una autoría compartida, entre Eileen y Jean Badovici, algo que sin duda propició que «con el tiempo la omisión del nombre de Gray se hiciera más frecuente cuando se mencionaba la casa E-1027» (Espegel, 2007: 108). Un hecho cuanto menos significativo, y que ha contribuido durante todo este tiempo a que un personaje como Eileen Gray permaneciera en el ostracismo de la historia del arte, la arquitectura y el diseño.

Esta actitud de Badovici tiene mucho que ver con la que posteriormente desenvuelve Le Corbusier. Tengamos presente que Gray llegó a establecer una relación cordial con Le Corbusier-aunque siempre resultó algo ambigua-por el tiempo que pasaba el arquitecto junto a 
Badovici, en diferentes lugares incluida su casa de Cap Martin. Sin embargo, entre ella y Le Corbusier no se fraguaría una amistad profunda, a pesar del tiempo que pasaban juntos. La propia biografía de Gray es explícita en esta cuestión, señalando que «Eileen's relationship with Le Corbusier was amiable but distant» (Adam, 1987: 309).

Esta relación se acabaría definitivamente en 1938, cuando Le Corbusier comenzaría a pintar, sin autorización, una serie de murales en la casa (Fig. 5). Algo que ella llegaría a considerar como «an act of a vandalism» (Adam, 1987: 311). Sin embargo, en este momento, Eileen no sólo se había distanciado de Badovici, sino que su relación sentimental estaba totalmente acabada, tanto que ella residía ya en una casa que construyó para sí misma en Castellar, una localidad a pocos kilómetros de Roquebrune hacia el interior. Ante estas circunstancias y recordando que la propiedad legal de la E1027 estaba a nombre de Jean Badovici, «no podía hacer nada para evitar que Le Corbusier «regalara» los murales a su propietario» (Marcos, 2011: 267).
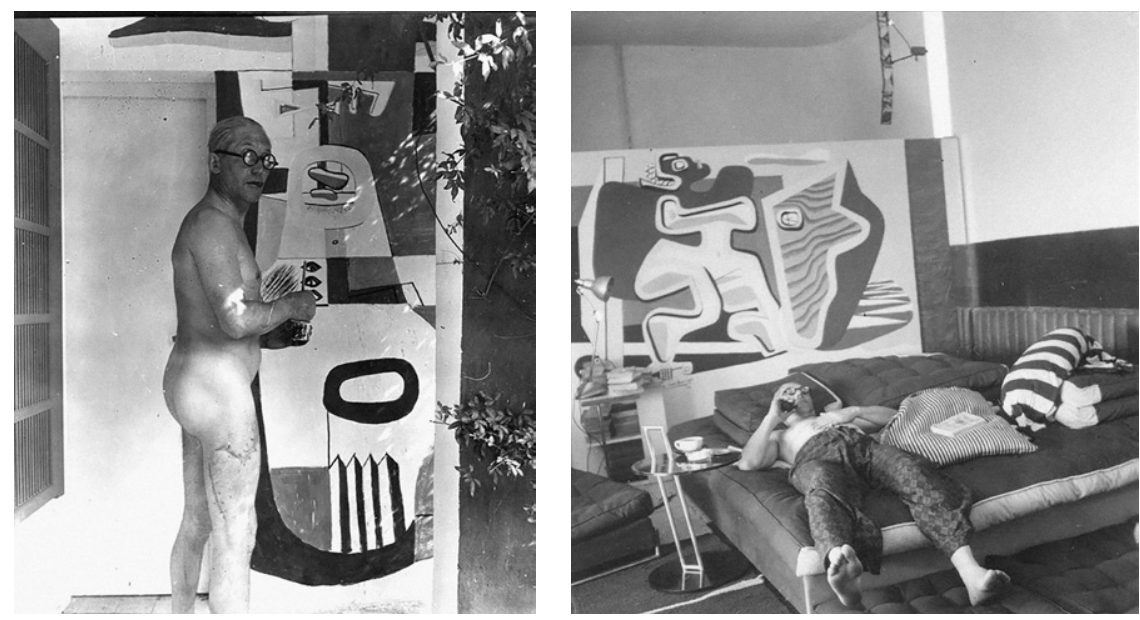

Fig. 5. Le Corbusier junto a unos de los murales en la villa E1027, s/f.

Posiblemente, una de las cuestiones más dolorosas respecto a estos murales sería, no el hecho de pintar un mural en sí mismo, sino la invasión que suponía el realizarlo en una casa que él no había diseñado. Recordemos que, tal y como hemos expuesto, tanto Le Corbusier como Eileen Gray y otros muchos arquitectos partícipes de lo denominado como Movimiento Moderno, consideraban cada construcción y cada diseño como una obra de arte total, esa visión arquitectónica del Gesamtkunstwerk de Wegner, que tan bien supo hacer 
suya la escuela de la Bauhaus. Por estas razones, el hecho que más disgustó a Gray fue que Le Corbusier no le pidiera permiso a ella, que había diseñado la casa, para pintar el mural. $\mathrm{Ni}$ tan siquiera su parecer sobre la posibilidad de hacerlo o no.

A pesar de este primer enfrentamiento, y que Le Corbusier supiera del disgusto que sus actos habían causado a la arquitecta, continuó con su intención de ampliar sus trabajos en la vivienda. Pintó varios murales más, pero «sin duda el más doloroso debió ser la ejecución del fresco de la entrada sobre otro que la propia Eileen Gray había ejecutado en el mismo parámetro» (Marcos, 2011 : 271). La postura de Badovici sobre las actuaciones de Le Corbusier, a pesar de conocer cuánto disgustaron a Eileen Gray, fue bastante ambigua, y es que, a pesar de todo «los murales tendrían un valor nada desdeñable que iría incrementándose con el paso del tiempo» (Marcos, 2011: 268), un hecho que repercutiría seriamente en la decisión de Badovici de seguir permitiendo las intervenciones de Le Corbusier sobre la E1027, muy a pesar de que «el tratamiento cromático y la potencia formal de los frescos poco o nada tienen que ver con la pureza y la sencillez formal de la E1027» (Marcos, 2011 : 274).

Pero los propósitos de Le Corbusier sobre la E1027, no quedaron sólo aquí. Era una casa que desde siempre le había maravillado; en ese sentido, nos recuerda Espegel que él «admiraba la villa E1027 y reconocía que con ella, Gray había conseguido distinguirse de sus contemporáneos» (2007: 129). Así, diez años más tarde de que pintara el primer fresco en las paredes de la casa, envió una carta a Badovici animándolo a reformar algunas estancias, e instándolo a eliminar algunas piezas del mobiliario que Gray había diseñado específicamente para cada lugar. Comenzaría una campaña de constante violación hacia el diseño original de la casa, tratando de asimilarla a los parámetros courbuserianos. Quería hacer suyo un diseño genial que no le pertenecía, y estaba dispuesto a hacer cualquier cosa por conseguir atribuirse aquella construcción.

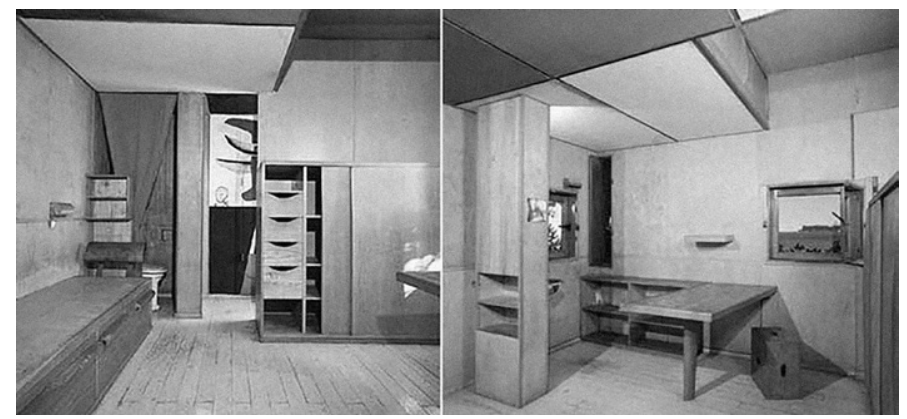

Fig. 6. Le Corbusier. Interior de Le Cabanon. 
Uno de los planteamientos más agresivos con el entorno de la casa, fue sin duda el proyecto de construcción de unas agrupaciones modulares de viviendas que denominaría Rob y Roq. Este proyecto, a diferencia de la composición horizontal de la villa diseñada por Gray, fue diseñado en perpendicular, maximizando el desnivel. Algo que, seguramente, fue una de las razones que hizo inviable el proyecto. Pero el fracaso del Rob y Roq no supuso desmotivación alguna para Le Corbusier, y seguiría sin cesar con su plan de colonizar el perímetro de la E-1027. Unos años más tarde, logró unos permisos para edificar a tan sólo veinte metros de la villa E1027, una pequeña construcción modular denominada Le Cabannon(Fig. 6). Un pequeño habitáculo en el que se reducía el espacio habitacional necesario, con el que «al imponer su visión desde arriba, estableció su dominio sobre el lugar de la casa de Gray. El cabannon no era más que una plataforma de observación» (Colomina cit. en Marcos, 2011 : 277). Posteriormente, la situación todavía se agravaría más, pues junto al Cabannon, el arquitecto lograría los permisos necesarios para construir el conjunto de las Unité de Camping.

Paradójicamente «el conjunto de intervenciones proyectadas por Le Corbusier en Cap-Martin evidencian una actitud de apropiación o colonización del lugar intentando crear un marco corbuseriano» (Marcos, 201 1: 280), pero el resultado final no logra en ningún caso crear ese marco, pues el conjunto construcciones que allí desarrolla, cuentan unas tipologías absolutamente alejadas del Estilo Internacional. Sin duda un hecho muy llamativo que parece confirmar que «El cabannon no se puede entender más que como patología. Nada de lo que hay en él responde a los cánones del dictado corbuseriano. [...] Todo lo que rodea las intervenciones de Le Corbusier en Cap-Martin parece inexplicable» (Marcos 201 1: 280-282).

Con todo, las intenciones de apropiarse de la Casa por parte de Le Corbusier no llegarían sólo hasta su perímetro. Tras la muerte de Jean Badovici, la vivienda pasó a ser propiedad de la hermana de éste, cuya vocación había sido la de ser monja de clausura en un convento de una localidad de Rumanía y necesariamente rechazó la herencia. Posteriormente, la casa salió a subasta pública y fue entonces cuando Le Corbusier animó a una amiga suya, Madame Schelbert, a pujar en su lugar. Finalmente, ésta consiguió adquirir la casa, y el arquitecto continuó pasando largas temporadas en la villa E1027, pero no sin antes prohibir a la propia Gray que retirara cualquier objeto de la Casa. Con este gesto, reconocía implícitamente la premisa con la que la propia arquitecta diseñó cada uno de los objetos con especial esmero. Paradójicamente, un tiempo después y tal vez por sentirse mal por sus acciones con Eileen, Le Corbusier le ofrecería dinero, pero ella siempre se negó a aceptarlo. La historia llegaría a su fin en agosto de 1965, cuando Le Corbusier se dispuso a bajar al mar, a tomar el baño y nadar como solía hacer habitualmente, pero nunca más regresó, ni tampoco se supo más de él. 
Sin embargo, y a pesar de todas las acciones que realizó Le Corbusier sobre la E1027, posiblemente, la que más perjudicaría a Eileen Gray y la difusión de su trayectoria como arquitecta, sería precisamente que en muchos de los textos en los que se trataron los famosos murales que ejecutó en las paredes de la casa, se obviaba el nombre de la arquitecta que la había diseñado. Con este simple gesto, que daba por hecho que las paredes de los murales eran las paredes de una obra arquitectónica diseñada por el mismo artista, la historiografía contribuiría a que Gray no tuviera, durante mucho tiempo, el reconocimiento que su talento merecía.

No podemos descuidar algunas cuestiones que nos parecen interesantes en cuanto a este enfrentamiento en el que se ven involucrados Le Corbusier y Eileen Gray, por las connotaciones de género que pueden percibirse. Posiblemente, uno de los aspectos que menos gustaría a Le Corbusier acerca de la arquitectura de Gray, y especialmente de la villa E1027, sería la forma en que la arquitecta supo desenvolverse dejando a un lado la concepción de Machine d'Habiter, porque para ella «la arquitectura debía estar al servicio del individuo, pero además debía relacionarse con todo su ser, con su experiencia corporal, con la gravedad de su materialidad» (Marcos, 2011: 291), incluso llegaría a afirmar que "a house is not a machine to live in» (Gray cit. en Marcos, 2011: 291). Es conveniente recordar que una de las grandes críticas que se ha hecho posteriormente al Estilo Internacional, del que Le Corbusier se convierte en uno de sus principales referentes, es precisamente la falta de comprensión y de sensibilidad con el lugar en el que se desarrolla, dejando a un lado no sólo tradiciones arquitectónicas específicas de cada sociedad, sino el propio territorio y su configuración orográfica natural. Frente a esto, observamos como «la E1027 se acomoda y hace suyo el lugar de muchas maneras, la casa no podría estar en otro sitio distinto de que está» (Marcos, 2011 : 293), y asimismo «el proyecto se utiliza como arma de distinción y de resistencia contra la uniformidad cartesiana moderna que niega toda tradición» (Espegel, 2010: 268).

Eileen Gray, a partir de los años treinta comenzaría a reducir el número de sus trabajos, en gran medida por el desencanto que vivía constantemente por la falta de reconocimiento en los diferentes campos en los que se adentró, pero muy especialmente en el de la arquitectura, hecho en el que influyó indudablemente su condición de mujer. Así, cuando estalló la Segunda Guerra Mundial, tuvo que salir de su casa de Castellar -posiblemente la construcción en la que más cómoda llegó a sentirse- y las circunstancias bélicas le impedieron regresar a un París inmerso en el conflicto. Llegó a plantearse regresar a Londres, pero convencida de que aquél no era su lugar decidió permanecer durante un tiempo en una pequeña localidad de Suiza. Acabada la Guerra, regresó a su apartamento de París, donde poco a poco, se iría apagando la llama vital de Eileen Gray hasta fallecer en su casa a los 
98 años de edad, dejando para la posteridad un legado profesional complejo en el que los límites entre arquitectura, interiorismo y mobiliario parecen homogeneizarse.

Ella, autora que se movió entre la sutileza sofisticada del Art Déco y la funcionalidad de la Bauhaus, fue sin duda un exponente de lo mejor de la arquitectura y el diseño del siglo $X X$. Sin prejuicios, supo moverse en las corrientes de la decoración a la par que asimilar los grandes logros de la estética del movimiento moderno, sin olvidar ni dejar de lado la íntima relación que tanto diseño como arquitectura entablan con las personas y sus formas de habitar, y por tanto, de vivir.

\section{Referencias y bibliografía}

AdAM, Peter (1987): Eileen Gray. Architect/Designer, London, Thames and Hundson.

CAmpelo Tenoira, Mariola (2012): «Lucia Moholy y Berenice Abbott: fotografía de arquitectura» en De Arte, num. 11, León, Publicaciones de la Universidad de León.

DACHS, Sandra y otros (2013): Eileen Gray, muebles y objetos, Barcelona: Polígrafa.

Espegel, Carmen (2007): Heroínas del espacio. Mujeres arquitectos en el Movimiento Moderno, Nobuko, Buenos Aires.

GaRner, Philiphe (1993): Eileen Gray designer and architect, London: Taschen.

HeCKer, Stefan y Christian F. Müller (1993): Eileen Gray. Works And Projects, Barcelona: Gustavo Gili.

HofmanN, Werner (1992): Los fundamentos del arte moderno, Barcelona: Península.

Marcos, Carlos Luis (201 1): «Crítica de género. E1027: Eileen Gray vs. Le Corbusier en Cap Martin», en Feminismo/s, 17, Alicante: Universidad de Alicante, pp. 259-295.

Moran, Lisa (2013): Eileen Gray: Architect, Designer, Painter, Dublin: Irish Musuem of Modern Art.

RAWSTHORN, Alice (2013): «Eileen Gray, Freed form Seclusion», en New York Times, 24 de febrero de 2013. [Consultado a 10-09-2015]: http://nyti.ms/Yr8ZWv 